\title{
Relative Homology Groups of Digital Images
}

\author{
Ozgur Ege E,* Ismet Karaca $^{2}$ and Meltem Erden Ege ${ }^{2}$ \\ ${ }^{1}$ Department of Mathematics, Celal Bayar University, 45140 Muradiye, Manisa, Turkey \\ ${ }^{2}$ Department of Mathematics, Ege University, 35100 Bornova, Izmir, Turkey
}

Received: 1 Sep. 2013, Revised: 29 Nov. 2013, Accepted: 30 Nov. 2013

Published online: 1 Sep. 2014

\begin{abstract}
In this paper we are interested in relative homology groups of digital images. Some properties of the Euler characteristics for digital images are given. We also present reduced homology groups for digital images. The main purpose is to obtain some differences between notions in digital topology and algebraic topology.
\end{abstract}

Keywords: Digital image, digital relative homology group, euler characteristics

\section{Introduction}

The fundamental group is a very valuable tool but it has an important deficiency. It is not enough to solve problems such as showing that $S^{3}$ and $S^{4}$ are not homeomorphic. Using the homology group of a simplicial complex, this difficulty could be overcome. Homology groups offer a different approach to hole counting. For instance, with this approach, the $n$-sphere has one $n$-dimensional hole and no $m$-dimensional holes for $m \neq n$.

The homology theory is the main field of algebraic topology. It plays a significant role in the classification problems of topological spaces. There are various approaches to the study of this theory. One of these is the simplicial homology. Homology groups are invariants from algebraic topology which are frequently used in digital image analysis and structural pattern recognition. They are related to the different n-dimensional holes, connected components, tunnels, cavities, etc., of a geometric object.

In 1927, Lefschetz defined the relative homology groups. They are important and useful for a number of reasons, principally for computational ones, since they fit into long exact sequences, which are powerful computational tools in homology. Moreover they help determine what part of an absolute homology group comes from which subspace. The relative homology is defined to compute the holes modulo some region of space in some applications.
Arslan et al. [1] introduce the simplicial homology groups of $n$-dimensional digital images. Moreover, they compute simplicial homology groups of $M S S_{18}$.

Diaz-Pernil et.al. [8] present a new solution for the homology groups of binary $2 \mathrm{D}$ image problem which is classical problem in homology theory which tries to calculate the number of connected components and the representative curves of the holes of these components from a given binary 2D image, by using membrane computing techniques.

Boxer, Karaca and Oztel [7] expand knowledge of the simplicial homology groups of digital images. They study the simplicial homology groups of certain minimal simple closed surfaces. They also extend an earlier definition of the Euler characteristics of digital image and compute the Euler characteristics of several digital surfaces.

Karaca and Ege [11] give some results related to the simplicial homology groups of $2 D$ digital images. They show that if a bounded digital image $X \subset \mathbb{Z}$ is nonempty and $\kappa$-connected, then its homology groups at the first dimension are a trivial group. In [11], it's proved that the homology groups of the operands of a wedge of digital images need not be additive.

Ege and Karaca [9] study the properties of the simplicial homology groups of digital images and investigate Eilenberg-Steenrod axioms for the simplicial homology groups of digital images.

This article is organized as follows. In Section 2, we give the general notions of digital images with $\kappa$ adjacency relations, digital homotopy groups and digital

\footnotetext{
*Corresponding author e-mail: ozgur.ege@cbu.edu.tr
} 
homology groups. In the next section we introduce the relative homology groups for digital images, give sample calculations and provide some important properties. In the section 4, we deal with properties of the Euler characteristics. In the last section we make some conclusions.

\section{Preliminaries}

A pair $(X, \kappa)$ is called a digital image, where $\mathbb{Z}$ is the set of integers, $X \subset \mathbb{Z}^{n}$ for some positive integer $n$ and $\kappa$ represents certain adjacency relation for the members of $X$. We give an adjacency relation which is used in the study of digital images. Let $l, n$ be positive integers such that $1 \leq l \leq n$ and two distinct points

$$
p=\left(p_{1}, p_{2}, \ldots, p_{n}\right), q=\left(q_{1}, q_{2}, \ldots, q_{n}\right)
$$

in $\mathbb{Z}^{n}$. If there are at most $l$ distinct coordinates $j$ for which $\left|p_{j}-q_{j}\right|=1$ and for all other coordinates $j$, $p_{j}=q_{j}$ then we say that $p$ and $q$ are $k_{l}$-adjacent [5]. The number of points $q \in \mathbb{Z}^{n}$ that are adjacent to a given point $p \in \mathbb{Z}^{n}$ are represented by a $k_{l}$-adjacency relation. In $\mathbb{Z}$, $k_{1}$-adjacent points are called 2-adjacent; in $\mathbb{Z}^{2}$, $k_{1}$-adjacent points and $k_{2}$-adjacent points are called 4 -adjacent and 8-adjacent; finally in $\mathbb{Z}^{3}, k_{1}, k_{2}$ and $k_{3}$-adjacent points are called 6 -adjacent, 18 -adjacent and 26-adjacent, respectively.

Let $\kappa$ be an adjacency relation defined on $\mathbb{Z}^{n}$. A $\kappa$-neighbor of $p \in \mathbb{Z}^{n}$ is a point of $\mathbb{Z}^{n}$ that is $\kappa$-adjacent to $p$. A digital image $X \subset \mathbb{Z}^{n}$ is $\kappa$-connected [10] if and only if for every pair of different points $x, y \in X$, there is a set $\left\{x_{0}, x_{1}, \ldots, x_{r}\right\}$ of points of a digital image $X$ such that $x=x_{0}, y=x_{r}$ and $x_{i}$ and $x_{i+1}$ are $\kappa$-neighbors where $i=0,1, \ldots, r-1$. A $\kappa$-component of a digital image $X$ is a maximal $\kappa$-connected subset of $X$. A set of the form $[a, b]_{\mathbb{Z}}=\{z \in \mathbb{Z} \mid a \leq z \leq b\}$ is a digital interval [2], where $a, b \in \mathbb{Z}$ with $a<b$.

Let $\left(X, \kappa_{0}\right) \subset \mathbb{Z}^{n_{0}}$ and $\left(Y, \kappa_{1}\right) \subset \mathbb{Z}^{n_{1}}$ be digital images. A function $f: X \longrightarrow Y$ is $\left(\kappa_{0}, \kappa_{1}\right)$-continuous [3], if for every $\kappa_{0}$-connected subset $U$ of $X, f(U)$ is a $\kappa_{1}$-connected subset of $Y$. In [3], it is shown that a function $f: X \longrightarrow Y$ is $\left(\kappa_{0}, \kappa_{1}\right)$-continuous if and only if for every $\kappa_{0}$-adjacent points $\left\{x_{0}, x_{1}\right\}$ of $X$, either $f\left(x_{0}\right)=f\left(x_{1}\right)$ or $f\left(x_{0}\right)$ and $f\left(x_{1}\right)$ are a $\kappa_{1}$-adjacent in $Y$.

In a digital image $(X, \kappa)$, a $(2, \kappa)$-continuous function $f:[0, m]_{\mathbb{Z}} \longrightarrow X$ such that $f(0)=x$ and $f(m)=y$ is called a digital $\kappa$-path [6] from $x$ to $y$. If $f(0)=f(m)$ then $f$ is a digital $\kappa$-loop and the point $f(0)$ is the base point of the loop $f$.

Definition 2.1. [4]. A simple closed $\kappa$-curve of $m \geq 4$ points in a digital image $X$ is a sequence $\{f(0), f(1), \ldots, f(m-1)\}$ of images of the $\kappa$-path $f:[0, m-1]_{\mathbb{Z}} \longrightarrow X$ such that $f(i)$ and $f(j)$ are $\kappa$-adjacent if and only if $j=i \pm \bmod m$.

Let $\left(X, \kappa_{0}\right) \subset \mathbb{Z}^{n_{0}}$ and $\left(Y, \kappa_{1}\right) \subset \mathbb{Z}^{n_{1}}$ be digital images. A function $f: X \longrightarrow Y$ is a $\left(\kappa_{0}, \kappa_{1}\right)$-isomorphism [6] if $f$ is $\left(\kappa_{0}, \kappa_{1}\right)$-continuous and bijective and also $f^{-1}: Y \longrightarrow X$ is $\left(\kappa_{1}, \kappa_{0}\right)$-continuous. For a digital image $(X, \kappa)$ and its subset $(A, \kappa)$, we call $(X, A)$ a digital image pair with $\kappa$ adjacency.

Definition 2.2. [3]. Let $X \in \mathbb{Z}^{n_{0}}$ and $Y \in \mathbb{Z}^{n_{1}}$ be digital images with $\kappa_{0}$-adjacency and $\kappa_{1}$-adjacency respectively. For two $\left(\kappa_{0}, \kappa_{1}\right)$-continuous functions $f, g: X \longrightarrow Y$, if there is a positive integer $m$ and a function $H: X \times[0, m]_{\mathbb{Z}} \longrightarrow Y$ such that

- for all $x \in X, H(x, 0)=f(x)$ and $H(x, m)=g(x)$;

- for all $x \in X$, the induced function $H_{x}:[0, m]_{\mathbb{Z}} \longrightarrow Y$ defined by

$$
H_{x}(t)=H(x, t) \quad \text { for all } t \in[0, m]_{\mathbb{Z}},
$$

is $\left(2, \kappa_{1}\right)$-continuous; and

- for all $t \in[0, m]_{\mathbb{Z}}$, the induced function $H_{t}: X \longrightarrow Y$ defined by

$$
H_{t}(x)=H(x, t) \quad \text { for all } x \in X
$$

is $\left(\kappa_{0}, \kappa_{1}\right)$-continuous, they are said to be digitally $\left(\kappa_{0}, \kappa_{1}\right)$-homotopic in $Y$ and this is denoted by $f \simeq_{\left(\kappa_{0}, \kappa_{1}\right)} g$. The function $H$ is called a digital $\left(\kappa_{0}, \kappa_{1}\right)$-homotopy between $f$ and $g$. The digital homotopy relation is equivalence relation among digitally continuous functions (see [3]).

Let $f: X \longrightarrow Y$ and $g: Y \longrightarrow X$ be $\left(\kappa_{0}, \kappa_{1}\right)$ and $\left(\kappa_{1}, \kappa_{0}\right)$ continuous functions respectively such that

$$
f \circ g \simeq_{\left(\kappa_{1}, \kappa_{1}\right)} 1_{Y} \text { and } g \circ f \simeq_{\left(\kappa_{0}, \kappa_{0}\right)} 1_{X}
$$

We say that $X$ and $Y$ have the same $\left(\kappa_{0}, \kappa_{1}\right)$-homotopy type and that $X$ and $Y$ are $\left(\kappa_{0}, \kappa_{1}\right)$-homotopy equivalent [3].

Definition 2.3. [3]. (i) A digital image $(X, \kappa)$ is said to be $\kappa$-contractible if its identity map is $(\kappa, \kappa)$-homotopic to a constant function $\bar{c}$ for some $c \in X$ where the constant function $\bar{c}: X \longrightarrow X$ is defined by $\bar{c}(x)=c$ for all $x \in X$.

(ii) Let $(X, A)$ be a digital image pair with $\kappa$-adjacency and $i: A \longrightarrow X$ be the inclusion function. $A$ is called a $\kappa$-retract of $X$ if and only if there is a $\kappa$-continuous function $r: X \longrightarrow A$ such that $r(a)=a$ for all $a \in A$. Then the function $r$ is called a $\kappa$-retraction of $X$ onto $A$.

(iii) A digital homotopy $H: X \times[0, m]_{\mathbb{Z}} \longrightarrow X$ is a deformation $\kappa$-retract if the induced map $H(-, 0)$ is the identity map $1_{X}$ and the induced map $H(-, m)$ is retraction of $X$ onto $H(X \times\{m\}) \subset X$. The set $H(X \times\{m\})$ is called a deformation $\kappa$-retract of $X$.

Definition 2.4. [12]. Let $(X, \kappa)$ be a digital image and $S$ be a set of nonempty subset $X$. Assume that the following hold: 
a) If $p$ and $q$ are distinct points of $s \in S$, then $p$ and $q$ are $\kappa$-adjacent.

b) If $s \in S$ and $\emptyset \neq t \subset s$, then $t \in S$.

Then the members of $S$ are called simplexes of $(X, \kappa)$. An $m$-simplex is a simplex $S$ such that $|S|=m+1$.

Let $P$ be a digital $m$-simplex. If $P^{\prime}$ is a nonempty proper subset of $P$, then $P^{\prime}$ is called a face of $P$. $\operatorname{Vert}(P)$ is used to denote the vertex set of $P$. A digital subcomplex $A$ of a digital simplicial complex $X$ with $\kappa$-adjacency is a digital simplicial complex [12] contained in $X$ with $\operatorname{Vert}(A) \subset \operatorname{Vert}(X)$.

Let $(X, \kappa)$ be a finite collection of digital $m$-simplices, $0 \leq m \leq d$ for some non-negative integer $d$. If the following statements hold then $(X, \kappa)$ is called a finite digital simplicial complex [1]:

- If $P$ belongs to $X$, then every face of $P$ also belongs to $X$.

- If $P, Q \in X$, then $P \cap Q$ is either empty or a common face of $P$ and $Q$.

The dimension of a digital simplicial complex $X$ is the largest integer $m$ such that $X$ has an $m$-simplex. $C_{q}^{\kappa}(X)$ is a free abelian group [1] with basis all digital $(\kappa, q)$-simplices in $X$.

Let $(X, \kappa) \subset \mathbb{Z}^{n}$ be a digital simplicial complex of dimension $m$. Boxer et.al. show that (see [7]) for all $q>m, C_{q}^{\kappa}(X)$ is a trivial group. The homomorphism $\partial_{q}: C_{q}^{\kappa}(X) \longrightarrow C_{q-1}^{\kappa}(X)$ defined (see [1]) by

$$
\partial_{q}(u)=\left\{\begin{array}{lr}
\sum_{i=0}^{q}(-1)^{i}<p_{0}, p_{1}, \ldots, \hat{p}_{i}, \ldots, p_{q}>, & q \leq m \\
0, & q>m
\end{array}\right.
$$

is called a boundary homomorphism, where $\hat{p}_{i}$ means delete the point $p_{i}$ and $u=<p_{0}, p_{1}, \ldots, p_{q}>$. In [1], Havana et.al. show that for all $1 \leq q \leq m$,

$$
\partial_{q-1} \circ \partial_{q}=0
$$

Definition 2.5. [1]. Let $(X, \kappa)$ be a digital simplicial complex.

(1) $Z_{q}^{\kappa}(X)=\operatorname{Ker} \partial_{q}$ is called the group of digital simplicial $q$-cycles.

(2) $B_{q}^{\kappa}(X)=\operatorname{Im} \partial_{q+1}$ is called the group of digital simplicial $q$-boundaries.

(3) $H_{q}^{\kappa}(X)=Z_{q}^{\kappa}(X) / B_{q}^{\kappa}(X)$ is called the $q$ th digital simplicial homology group.

Theorem 2.6. [7]. Let $(X, \kappa)$ be a directed digital simplicial complex of dimension $m$. $q \geq 0$.

- $H_{q}^{\kappa}(X)$ is a finitely generated abelian group for every

- $H_{q}^{\kappa}(X)$ is a trivial group for all $q>m$.

- $H_{m}^{\kappa}(X)$ is a free abelian group, possible zero.

Theorem 2.7. [1]. If $(X, \kappa)$ is a single point digital image, then

$$
H_{q}^{\kappa}=\left\{\begin{array}{l}
\mathbb{Z}, q=0 \\
0, q \neq 0
\end{array}\right.
$$

Theorem 2.8. [7]. For each $q \geq 0, H_{q}^{\kappa}$ is a covariant functor from the category of digital simplicial complexes and simplicial maps to the category of abelian groups.

Corollary 2.9. [1]. If $f:\left(X, \kappa_{1}\right) \longrightarrow\left(Y, \kappa_{2}\right)$ is a digitally $\left(\kappa_{1}, \kappa_{2}\right)$-isomorphism, then $f_{*}: H_{q}^{\kappa_{1}}(X) \longrightarrow H_{q}^{\kappa_{2}}(Y)$ is a group isomorphism.

\section{Digital relative homology groups}

Let $(A, \kappa)$ be a digital subcomplex of the digital simplicial complex $(X, \kappa)$. Then the chain group $C_{q}^{\kappa}(A)$ is a subgroup of the chain group $C_{q}^{\kappa}(X)$. The quotient group

$$
C_{q}^{\kappa}(X, A)=C_{q}^{\kappa}(X) / C_{q}^{\kappa}(A)
$$

is called the group of relative chains of $X$ modulo $A$. The boundary operator

$$
\partial_{q}: C_{q}^{\kappa}(A) \longrightarrow C_{q-1}^{\kappa}(A)
$$

is the restriction of the boundary operator on $C_{q}^{\kappa}(X)$. It induces a homomorphism

$$
C_{q}^{\kappa}(X, A) \longrightarrow C_{q-1}^{\kappa}(X, A)
$$

of the relative chain groups and this is also denoted by $\partial_{q}$.

Definition 3.1. [9]. Let $(A, \kappa)$ be a digital subcomplex of the digital simplicial complex $(X, \kappa)$.

- $Z_{q}^{\kappa}(X, A)=\operatorname{Ker} \partial_{q}$ is called the group of digital relative simplicial $q$-cycles.

- $B_{q}^{\kappa}(X, A)=\operatorname{Im} \partial_{q+1}$ is called the group of digital relative simplicial $q$-boundaries.

- $H_{q}^{\kappa}(X, A)=Z_{q}^{\kappa}(X, A) / B_{q}^{\kappa}(X, A)$ is called the $q$ th digital relative simplicial homology group.

Example 3.2. Let $X=[0,1]_{\mathbb{Z}}$ be the digital interval and $A$ be a single point digital image $\{0\}$. Let's determine $H_{q}^{2}(X, A)$ for all $q \geq 0$. For $q=0$,

$$
C_{0}^{2}(X) \cong \mathbb{Z} \oplus \mathbb{Z}, C_{0}^{2}(A) \cong \mathbb{Z} \text { and } C_{0}^{2}(X, A) \cong \mathbb{Z} .
$$

For $q=1$,

$$
C_{1}^{2}(X) \cong \mathbb{Z}, C_{1}^{2}(A) \cong 0 \text { and } C_{1}^{2}(X, A) \cong \mathbb{Z} .
$$

Since there are no digital $(2, q)$-simplexes of dimension 2 or higher, then

$$
C_{q}^{2}(X, A)=0
$$

and accordingly $H_{q}^{2}(X, A)=0$ for all $q \geq 2$.

Now consider the following short sequence:

$$
0 \stackrel{\partial_{2}}{\rightarrow} C_{1}^{2}(X, A) \stackrel{\partial_{1}}{\rightarrow} C_{0}^{2}(X, A) \stackrel{\partial_{0}}{\rightarrow} 0 .
$$

There are obvious results such that

$$
\operatorname{Im} \partial_{2}=0 \text { and } \operatorname{Ker} \partial_{0} \cong \mathbb{Z} .
$$


The elements of $C_{1}^{2}(X, A)$ are of the form

$$
z=g \cdot[0,1]_{\mathbb{Z}}+C_{1}^{2}(A), \quad g \in \mathbb{Z} .
$$

Since

$$
\begin{aligned}
\partial_{1}(z) & =\partial_{1}\left(g \cdot[0,1]_{\mathbb{Z}}+C_{1}^{2}(A)\right) \\
& =g \cdot[1]_{\mathbb{Z}}-g \cdot[0]_{\mathbb{Z}} \\
& =g \cdot[1]_{\mathbb{Z}}+C_{0}^{2}(A),
\end{aligned}
$$

we get $\operatorname{Im} \partial_{1} \cong \mathbb{Z}$. As a result, we have $H_{0}^{2}(X, A)=0$.

By the First Isomorphism Theorem,

$$
\mathbb{Z} / \operatorname{Ker} \partial_{1} \cong \operatorname{Im} \partial_{1} \cong \mathbb{Z} \Rightarrow \operatorname{Ker} \partial_{1}=0 .
$$

Consequently, $H_{1}^{2}(X, A)=0$. Thus we have $H_{q}^{2}(X, A)=0$ for all $q \geq 0$.

Ege and Karaca [9] show that the long sequence

$$
\ldots \rightarrow H_{q}^{\kappa}(A) \stackrel{i_{*}}{\rightarrow} H_{q}^{\kappa}(X) \stackrel{p_{*}}{\rightarrow} H_{q}^{\kappa}(X, A) \stackrel{\partial_{*}}{\rightarrow} H_{q-1}^{\kappa}(A) \rightarrow \ldots
$$

is exact, where $i: A \longrightarrow X$ and $p: X \longrightarrow(X, A)$ are inclusion maps. This is called exactness axiom.

We now give some significant results about the relative simplicial homology groups of digital images.

Proposition 3.3. Let $(X, \kappa)$ be a digital image and $A \subset X$. If $A$ is empty set,

$$
H_{q}^{\kappa}(X, A) \cong H_{q}^{\kappa}(X)
$$

for all $q \geq 0$.

Proof. Since $A$ is empty, for all $q \geq 0$ we have $H_{q}^{\kappa}(A)=0$. By the long sequence

$$
\ldots \rightarrow H_{q}^{\kappa}(A) \stackrel{i_{*}}{\rightarrow} H_{q}^{\kappa}(X) \stackrel{p_{*}}{\rightarrow} H_{q}^{\kappa}(X, A) \stackrel{\partial_{*}}{\rightarrow} H_{q-1}^{\kappa}(A) \rightarrow \ldots,
$$

for all $q \geq 0$, we have a short sequence

$$
0 \rightarrow H_{q}^{\kappa}(X) \stackrel{p_{*}}{\rightarrow} H_{q}^{\kappa}(X, A) \rightarrow 0 .
$$

As a result, $p_{*}$ is an isomorphism and so

$$
H_{q}^{\kappa}(X) \cong H_{q}^{\kappa}(X, A) .
$$

Proposition 3.4. If $X=\bigcup_{j} X_{j}$ is a union of $\kappa$-connected components, then

$$
H_{q}^{\kappa}(X, A) \cong \bigoplus_{j} H_{q}^{\kappa}\left(X_{j}, A_{j}\right)
$$

where $A_{j}=A \cap X_{j}$.

Proof. The proof is the same as in Algebraic Topology.

Theorem 3.5. If $X$ is $\kappa$-connected and $A$ is nonempty $\kappa$ connected digital image, then

$$
H_{0}^{\kappa}(X, A)=0 .
$$

Proof. Consider the following long exact sequence:

$$
\ldots \stackrel{\partial_{2}}{\rightarrow} C_{1}^{\kappa}(X, A) \stackrel{\partial_{1}}{\rightarrow} C_{0}^{\kappa}(X, A) \stackrel{\partial_{0}}{\rightarrow} 0 .
$$

It's clear that $\operatorname{Ker} \partial_{0}=C_{0}^{\mathcal{K}}(X, A)$. The elements of $C_{1}^{\mathcal{K}}(X, A)$ are of the form

$$
z=g \cdot \sum_{j}<u_{j} u_{j+1}>+C_{1}^{\kappa}(A), \quad g \in \mathbb{Z}
$$

where $u_{j}$ is an arbitrary point of $X,<u_{j} u_{j+1}>$ is a digital 1-simplex in $X$ and

$$
C_{1}^{\kappa}(A)=\left\{\alpha<u_{j}>: \alpha \in \mathbb{Z}, j \in J\right\} .
$$

Since

$$
\begin{aligned}
\partial_{1}(z) & =\partial_{1}\left(g \cdot \sum_{j}<u_{j} u_{j+1}>+C_{1}^{\kappa}(A)\right) \\
& =g \cdot \sum_{j}<u_{j}>+C_{0}^{\kappa}(A),
\end{aligned}
$$

$\operatorname{Im} \partial_{1}=C_{0}^{\kappa}(X, A)$. Therefore, we have $H_{0}^{\kappa}(X, A)=0 . \square$

Proposition 3.6. If $A=\left\{x_{0}\right\}$ is a single point digital image, $H_{q}^{\kappa}(X, A) \cong H_{q}^{\kappa}(X)$ for $q>0$.

Proof. From Theorem 2.7, we know that $H_{q}^{\kappa}(A)=0$ for $q>0$. By the long sequence

$$
\ldots \rightarrow H_{q}^{\kappa}(A) \rightarrow H_{q}^{\kappa}(X) \rightarrow H_{q}^{\kappa}(X, A) \stackrel{\partial_{*}}{\rightarrow} H_{q-1}^{\kappa}(A) \rightarrow \ldots,
$$

for all $q>0$, we have a short sequence

$$
0 \rightarrow H_{q}^{\kappa}(X) \stackrel{p_{*}}{\rightarrow} H_{q}^{\kappa}(X, A) \rightarrow 0 .
$$

So $p_{*}$ is an isomorphism and we get

$$
H_{q}^{\kappa}(X) \cong H_{q}^{\kappa}(X, A) .
$$

Proposition 3.7. For any digital image $(X, \kappa)$,

$$
H_{q}^{\kappa}(X, X)=0
$$

for all $q \geq 0$.

Proof. Consider the exact digital homology sequence for $(X, X)$. It's clear that the inclusion map $i:(X, \kappa) \longrightarrow(X, \kappa)$ which is identity, induces identity homomorphism $i_{*}: H_{q}^{\kappa}(X) \longrightarrow H_{q}^{\kappa}(X)$ for all $q \geq 0$. For this reason, we get the required result. $\square$

Proposition 3.8. If $A \subset X$ is a deformation $\kappa$-retract, then $H_{q}^{\kappa}(X, A)=0$ for all $q \geq 0$.

Proof. Let $A$ be a deformation $\kappa$-retract of $X$. Then the inclusion map $i: A \longrightarrow X$ is a $(\kappa, \kappa)$-homotopy equivalence and the retract $r: X \longrightarrow A$ is its $(\kappa, \kappa)$-homotopy inverse, i.e.,

$$
i \circ r \simeq_{(\kappa, \kappa)} 1_{X} \text { and } r \circ i \simeq_{(\kappa, \kappa)} 1_{A} .
$$


Therefore $i_{*}: H_{q}^{\kappa}(A) \longrightarrow H_{q}^{\kappa}(X)$ is an $(\kappa, \kappa)$-isomorphism for all $q \geq 0$. From the following long exact sequence

$$
\ldots \rightarrow H_{q}^{\kappa}(A) \stackrel{i_{*}}{\rightarrow} H_{q}^{\kappa}(X) \stackrel{j_{*}}{\rightarrow} H_{q}^{\kappa}(X, A) \stackrel{\partial_{\text {* }}}{\rightarrow} H_{q-1}^{\kappa}(A) \rightarrow \ldots
$$

$j_{*}: H_{q}^{\kappa}(X) \longrightarrow H_{q}^{\kappa}(X, A)$ must be a zero map. By exactness,

$$
H_{q}^{\kappa}(X, A)=\operatorname{Ker} \partial_{*}=\operatorname{Im} j_{*}=0 .
$$

\section{Example 3.9. Let}

$$
X=\left\{p_{0}=(0,0), p_{1}=(1,0), p_{2}=(1,1)\right\} \subset \mathbb{Z}^{2}
$$

be a digital image with 8 -adjacency. Boxer et.al. [7] show that

$$
H_{q}^{8}(X)=\left\{\begin{array}{l}
\mathbb{Z}, q=0 \\
0, q \neq 0
\end{array}\right.
$$

Let $A=\left\{p_{0}\right\}$ be a single point digital image. By Theorem 2.7, we have

$$
H_{q}^{8}(A)=\left\{\begin{array}{l}
\mathbb{Z}, q=0 \\
0, q \neq 0
\end{array}\right.
$$

Define a map $H: X \times[0,2]_{\mathbb{Z}} \longrightarrow X$ by the following.

$$
\left\{\begin{array}{lr}
H(x, 0)=x, & t=0 \\
H\left(p_{0}, 1\right)=H\left(p_{2}, 1\right)=p_{0}, H\left(p_{1}, 1\right)=p_{1}, & t=1 \\
H\left(p_{0}, 2\right)=H\left(p_{1}, 2\right)=p_{0}, & t=2
\end{array}\right.
$$

where $x \in X$ and $t \in[0,2]_{\mathbb{Z}}$. It's clear that this map is a 8deformation retract of $X$ to $A$. For $q \geq 2$, it's obvious that

$$
H_{q}^{8}(X, A)=0 .
$$

So we have the following short sequence:

$$
0 \stackrel{i}{\rightarrow} H_{1}^{8}(X, A) \stackrel{j}{\rightarrow} \mathbb{Z} \stackrel{k}{\rightarrow} \mathbb{Z} \rightarrow H_{0}^{8}(X, A) \rightarrow 0 .
$$

By Theorem 3.5, we get $H_{0}^{8}(X, A)=0$. From the First Isomorphism Theorem and the fact that $\operatorname{Ker} j=\operatorname{Im} i=0$,

$$
H_{1}^{8}(X, A) / \operatorname{Ker} j \cong \operatorname{Im} j \cong \operatorname{Ker} k \cong 0 \Rightarrow H_{1}^{8}(X, A)=0 .
$$

As a result, we have $H_{q}^{8}(X, A)=0$ for all $q \geq 0$.

Theorem 3.10. Let $(X, A)$ be a digital image pair with $\kappa$ adjacency such that $(A, \kappa)$ is a $\kappa$-retract of $(X, \kappa)$. Then

$$
H_{q}^{\kappa}(X) \cong H_{q}^{\kappa}(A) \oplus H_{q}^{\kappa}(X, A) .
$$

Proof. Since $(A, \kappa)$ is a $\kappa$-retract of $(X, \kappa), r \circ i=1_{(A, \kappa)}$ where $i:(A, \kappa) \longrightarrow(X, \kappa)$ and $j:(X, \emptyset) \longrightarrow(X, A)$ are inclusion maps and $r:(X, \kappa) \longrightarrow(A, \kappa)$ is a $\kappa$-retraction map. So $r_{*} \circ i_{*}=1_{H_{q}^{K}(A)}$ and $i_{*}$ is a monomorphism. Consider the exactness of the homology sequence of $(X, A)$

$\ldots \rightarrow H_{q}^{\kappa}(X, A) \stackrel{\partial_{*}}{\rightarrow} H_{q-1}^{\kappa}(A) \stackrel{i_{*}}{\rightarrow} H_{q-1}^{\kappa}(X) \stackrel{j_{*}}{\rightarrow} H_{q-1}^{\kappa}(X, A) \stackrel{\partial_{*}}{\rightarrow} \ldots$ $\partial_{*}$ is the trivial map because Ker $i_{*}=0$. Thus $j_{*}$ is an epimorphism. Since Ker $j_{*}=\operatorname{Im} i_{*}=H_{q-1}^{\kappa}(A)$, we have

$$
\begin{aligned}
H_{q-1}^{\kappa}(X) / \operatorname{Ker} j_{*} & =H_{q-1}^{\kappa}(X) / H_{q-1}^{\kappa}(A) \\
& \cong H_{q-1}^{\kappa}(X, A) \\
& \cong \operatorname{Im} j_{*}
\end{aligned}
$$

by the First Isomorphism Theorem. As a result, $j_{*}$ induces a $(\kappa, \kappa)$-isomorphism. $\square$

\section{Example 3.11. Let}

$$
M S C_{8}^{\prime}=\{(1,0),(0,1),(-1,0),(0,-1)\} \subset \mathbb{Z}^{2}
$$

and

$$
\begin{gathered}
M S C_{4}=\{(1,0),(1,1),(0,1),(-1,1),(-1,0),(-1,-1), \\
(0,-1),(1,-1)\} \subset \mathbb{Z}^{2}
\end{gathered}
$$

be digital images with 8-adjacency (see Figure 1).
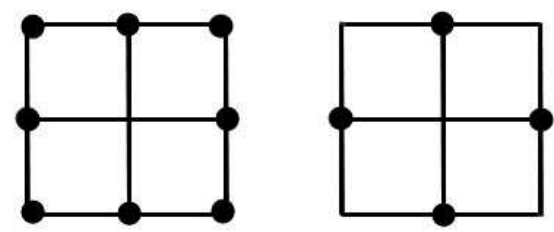

Fig. 1: $M S C_{4}$ and $M S C_{8}^{\prime}$

$M S C_{8}^{\prime}$ is digital 8-retract of $M S C_{4}$. Since $M S C_{8}^{\prime}$ and $M S C_{4}$ are digital simple closed 8-curve,

$$
H_{q}^{8}\left(M S C_{4}\right)=H_{q}^{8}\left(M S C_{8}^{\prime}\right)=\left\{\begin{array}{l}
\mathbb{Z}, q=0,1 \\
0, q \neq 0,1
\end{array}\right.
$$

(see [1]). Consider the following long exact homology sequence:

$$
\begin{gathered}
\cdots \rightarrow H_{2}^{8}\left(M S C_{8}^{\prime}\right) \rightarrow H_{2}^{8}\left(M S C_{4}\right) \rightarrow H_{2}^{8}\left(M S C_{4}, M S C_{8}^{\prime}\right) \\
\stackrel{\partial}{\rightarrow} H_{1}^{8}\left(M S C_{8}^{\prime}\right) \stackrel{i_{*}}{\rightarrow} H_{1}^{8}\left(M S C_{4}\right) \stackrel{j_{*}}{\rightarrow} H_{1}^{8}\left(M S C_{4}, M S C_{8}^{\prime}\right) \\
\stackrel{\partial}{\rightarrow} H_{0}^{8}\left(M S C_{8}^{\prime}\right) \stackrel{i_{*}}{\rightarrow} H_{0}^{8}\left(M S C_{4}\right) \stackrel{j_{*}}{\rightarrow} H_{0}^{8}\left(M S C_{4}, M S C_{8}^{\prime}\right) \rightarrow 0
\end{gathered}
$$

So we get

$$
0 \rightarrow \mathbb{Z} \stackrel{i_{*}}{\rightarrow} \mathbb{Z} \stackrel{j_{*}}{\rightarrow} H_{1}^{8}\left(M S C_{4}, M S C_{8}^{\prime}\right) \stackrel{\partial}{\rightarrow} \mathbb{Z} \stackrel{i_{*}}{\rightarrow} \mathbb{Z} \stackrel{j_{*}}{\rightarrow} 0
$$

By this exact sequence we have $H_{1}^{8}\left(M S C_{4}, M S C_{8}^{\prime}\right)=0$. As a result, for all $q \geq 0$

$$
H_{q}^{8}\left(M S C_{4}\right) \cong H_{q}^{8}\left(M S C_{8}^{\prime}\right) \oplus H_{q}^{8}\left(M S C_{4}, M S C_{8}^{\prime}\right)
$$


We now calculate the relative homology groups of $\left(M S S_{6}^{\prime}, A\right)$ where

$$
A=\left\{c_{0}, c_{1}, c_{2}, c_{3}\right\} \subset M S S_{6}^{\prime}
$$

has 6-adjacency.

Example 3.12. Let $\mathrm{MSS}_{6}^{\prime}=\left\{c_{0}=(0,0,0), c_{1}=\right.$ $(1,0,0), c_{2}=(1,1,0), c_{3}=(0,1,0), c_{4}=(0,0,1), c_{5}=$ $\left.(1,0,1), c_{6}=(1,1,1), c_{7}=(0,1,1)\right\}$ (see Figure 2$)$.

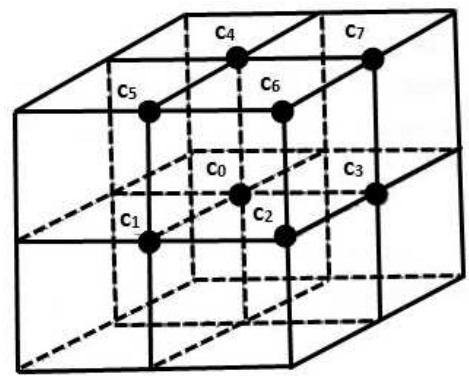

Fig. 2: $\mathrm{MSS}_{6}^{\prime}$

Its homology groups are [7]

$$
H_{q}^{6}\left(M S S_{6}^{\prime}\right)= \begin{cases}\mathbb{Z}, & q=0 \\ \mathbb{Z}^{5}, & q=1 \\ 0, & q \neq 0,1\end{cases}
$$

Since $A$ is digital simple closed 4-curve, we have

$$
H_{q}^{4}(A)=\left\{\begin{array}{l}
\mathbb{Z}, q=0,1 \\
0, q \neq 0,1
\end{array}\right.
$$

(see [1]). Consider the following long exact homology sequence:

$$
\begin{gathered}
\cdots \rightarrow H_{q+1}^{4}\left(M S S_{6}^{\prime}, A\right) \stackrel{\delta}{\rightarrow} H_{q}^{4}(A) \stackrel{i_{*}}{\rightarrow} H_{q}^{6}\left(M S S_{6}^{\prime}\right) \\
\stackrel{j_{*}}{\rightarrow} H_{q}^{4}\left(M S S_{6}^{\prime}, A\right) \rightarrow \ldots
\end{gathered}
$$

Then we have

$$
\begin{gathered}
0 \stackrel{p_{*}}{\rightarrow} H_{2}^{4}\left(M_{S S_{6}^{\prime}}^{\prime} A\right) \stackrel{\delta_{2}}{\rightarrow} \mathbb{Z} \stackrel{i_{*}}{\rightarrow} \mathbb{Z}^{5} \stackrel{j_{*}}{\rightarrow} H_{1}^{4}\left(M_{S S_{6}^{\prime}}^{\prime}, A\right) \stackrel{\delta_{1}}{\rightarrow} \mathbb{Z} \stackrel{k_{*}}{\rightarrow} \mathbb{Z} \\
\stackrel{l_{*}}{\rightarrow} H_{0}^{4}\left(M S S_{6}^{\prime}, A\right) \stackrel{\delta_{0}}{\rightarrow} 0 .
\end{gathered}
$$

By this exact sequence we have some conclusions:

- $H_{2}^{4}\left(M_{S S}^{\prime}, A\right)=0$ because $\operatorname{Im} p_{*}=0=\operatorname{Ker} \delta_{2}$ and from the First Isomorphism Theorem, we get

$$
H_{2}^{4}\left(M S S_{6}^{\prime}, A\right) / \operatorname{Ker} \delta_{2} \cong \operatorname{Im} \delta_{2}=\operatorname{Ker} i_{*}=0
$$

- Since Ker $\delta_{0}=H_{0}^{4}\left(M S S_{6}^{\prime}, A\right)=\operatorname{Im} l_{*}, \operatorname{Im} k_{*}=\operatorname{Ker} l_{*}=\mathbb{Z}$ we have

$$
H_{0}^{4}\left(M S S_{6}^{\prime}, A\right)=0
$$

- Using the exact sequence, we get

$$
\begin{gathered}
H_{0}^{4}\left(M S S_{6}^{\prime}, A\right) / \operatorname{Ker} \delta_{1} \cong \operatorname{Im} \delta_{1}=\operatorname{Ker} k_{*}=0 \\
\Rightarrow H_{0}^{4}\left(M S S_{6}^{\prime}, A\right)=\operatorname{Ker} \delta_{1}=\operatorname{Im} j_{*} .
\end{gathered}
$$

Similarly, since $\operatorname{Ker} j_{*}=\operatorname{Im} i_{*}=\mathbb{Z}^{5}$ we have

$$
\mathbb{Z}^{5} / \operatorname{Ker} j_{*} \cong \operatorname{Im} j_{*} \Rightarrow \operatorname{Im} j_{*}=0
$$

and consequently $H_{1}^{4}\left(M_{S S_{6}^{\prime}}^{\prime}, A\right)=0$.

As a result, for all $q \geq 0$, we have $H_{q}^{4}\left(M_{S S}^{\prime}, A\right)=0$.

We define a digital version of the real projective line $\mathbb{R} P^{1}$ via quotient map from $M S C_{4}$ with antipodal points. Denote the digital projective plane by $\mathbb{Z} P^{1}$. Let

$$
\begin{aligned}
M S C_{4}= & \left\{c_{0}=(-1,-1), c_{1}=(0,-1), c_{2}=(1,-1),\right. \\
c_{3}= & (1,0), c_{4}=(1,1), c_{5}=(0,1), c_{6}=(-1,1), \\
& \left.c_{7}=(-1,0)\right\} .
\end{aligned}
$$

If we take the quotient map $q: M S C_{4} \longrightarrow M S C_{4} / x \sim-x$ where $-x$ is the antipodal point of $x \in M S C_{4}$, then we have the digital projective line $\mathbb{Z} P^{1}$ (see Figure 3 ).

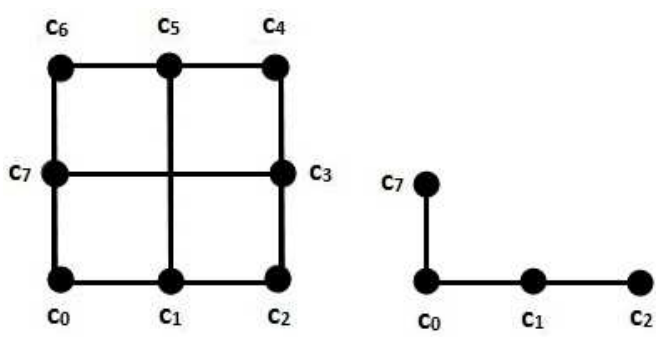

Fig. 3: $M S C_{4}$ and the Digital Projective Line $\mathbb{Z} P^{1}$

Define a map $H: \mathbb{Z} P^{1} \times[0,2]_{\mathbb{Z}} \longrightarrow \mathbb{Z} P^{1}$ by the following.

$$
\left\{\begin{array}{lr}
H(c, 0)=c, & t=0 \\
H\left(c_{7}, 1\right)=c_{0}, H\left(c_{2}, 1\right)=c_{1}, & t=1 \\
H\left(c_{1}, 2\right)=c_{0}, & t=2
\end{array}\right.
$$

where $c \in \mathbb{Z} P^{1}$. It's clear that this map is a 8-deformation retract of $\mathbb{Z} P^{1}$. So $\mathbb{Z} P^{1}$ is 8 -contractible image. As a result, $\mathbb{Z} P^{1}$ has the same digital homology groups as a single point image.

We want to calculate the relative homology groups of $\left(M S C_{4}, \mathbb{Z} P^{1}\right)$ where $\mathbb{Z} P^{1} \subset M S C_{4}$ is the digital projective line.

Example 3.13. Digital homology groups of $\mathbb{Z} P^{1}$ are

$$
H_{q}^{4}\left(\mathbb{Z} P^{1}\right)=\left\{\begin{array}{l}
\mathbb{Z}, q=0 \\
0, q \neq 0
\end{array}\right.
$$


Consider the following long exact homology sequence:

$$
\begin{gathered}
\cdots \rightarrow H_{q}^{4}\left(\mathbb{Z} P^{1}\right) \stackrel{i_{*}}{\rightarrow} H_{q}^{4}\left(M S C_{4}\right) \stackrel{j_{*}}{\rightarrow} H_{q}^{4}\left(M S C_{4}, \mathbb{Z} P^{1}\right) \\
\stackrel{\delta}{\rightarrow} H_{q-1}^{4}\left(\mathbb{Z} P^{1}\right) \rightarrow \ldots
\end{gathered}
$$

Then we have

$$
\begin{gathered}
0 \stackrel{i_{2}}{\rightarrow} \mathbb{Z} \stackrel{p_{2}}{\rightarrow} H_{1}^{4}\left(M S C_{4}, \mathbb{Z} P^{1}\right) \stackrel{\partial_{1}}{\rightarrow} \mathbb{Z} \stackrel{i_{1}}{\rightarrow} \mathbb{Z} \\
\stackrel{p_{1}}{\rightarrow} H_{0}^{4}\left(M S C_{4}, \mathbb{Z} P^{1}\right) \stackrel{\partial_{0}}{\rightarrow} 0 .
\end{gathered}
$$

By this exact sequence we have some conclusions:

- Since $\operatorname{Im} i_{2}=0=\operatorname{Ker} p_{2}$ and from the First Isomorphism Theorem, we have

$$
H_{1}^{4}\left(M S C_{4}, \mathbb{Z} P^{1}\right)=\mathbb{Z}
$$

- It's clear that $H_{0}^{4}\left(M S C_{4}, \mathbb{Z} P^{1}\right)=\operatorname{Ker} \partial_{0}$. By the exact sequence and the First Isomorphism Theorem, we have

$$
\mathbb{Z} / \operatorname{Ker} p_{1} \cong \operatorname{Im} p_{1}=0
$$

So we get $H_{0}^{4}\left(M S C_{4}, \mathbb{Z} P^{1}\right)=0$. As a result,

$$
H_{q}^{4}\left(M S C_{4}, \mathbb{Z} P^{1}\right)=\left\{\begin{array}{l}
\mathbb{Z}, q=1 \\
0, q \neq 1 .
\end{array}\right.
$$

If we make a little change the digital simplicial homology, we get a new concept which is reduced digital homology. The only difference between the two homologies lies on the group $H_{0}^{\kappa}(X)$.

Definition 3.14. [12]. Let $(X, \kappa)$ be a digital simplicial complex. We define a homomorphism $\varepsilon: C_{0}^{\kappa}(X) \longrightarrow \mathbb{Z}$ by putting $\varepsilon(v)=1$ for each vertex $v$ of $X$ and extending linearly to $C_{0}^{\kappa}(X)$. This homomorphism, called augmentation map, is onto. If $\partial_{1}: C_{1}^{\mathcal{K}}(X) \longrightarrow C_{0}^{\mathcal{K}}(X)$ is the boundary homomorphism, then $\varepsilon \circ \partial_{1}=0$.

$$
\widetilde{H_{0}^{\kappa}}(X)=\frac{\operatorname{Ker} \varepsilon}{\operatorname{Im} \partial_{1}}
$$

is called the zero dimensional reduced digital homology group of $(X, \kappa)$. If we put $\widetilde{H_{p}^{\kappa}}(X)=H_{p}^{\kappa}(X)$ for each $p>1$, then

$$
\left\{\widetilde{H_{i}^{K}}(X), i=0,1, \ldots\right\}
$$

are called the reduced digital homology groups of $(X, \kappa)$. There is an induced inclusion map $\widetilde{H_{0}^{\kappa}}(X) \longrightarrow H_{0}^{\kappa}(X)$ because $\operatorname{Im} \partial_{1} \subset \operatorname{Ker} \varepsilon \subset C_{0}^{\kappa}(X)$.

Theorem 3.15. [12]. For a digital simplicial complex $(X, \kappa)$, there are the following formulas which are related to reduced homology groups :

$$
\begin{gathered}
H_{0}^{\kappa}(X) \cong \widetilde{H_{0}^{\kappa}}(X) \oplus \mathbb{Z}, \\
H_{p}^{\kappa}(X)=\widetilde{H_{p}^{\kappa}}(X), \quad p \geq 1 .
\end{gathered}
$$

Proof. Since the sequence

$$
0 \rightarrow \operatorname{Ker} \varepsilon \rightarrow C_{0}^{\kappa}(X) \stackrel{\varepsilon}{\rightarrow} \mathbb{Z} \rightarrow 0
$$

is exact,

$$
0 \rightarrow \frac{\operatorname{Ker} \varepsilon}{\operatorname{Im} \partial_{1}} \rightarrow \frac{C_{0}^{\kappa}(X)}{\operatorname{Im} \partial_{1}} \stackrel{\varepsilon}{\rightarrow} \mathbb{Z} \rightarrow 0
$$

is also exact. It follows from $\mathbb{Z}$ is free that the above sequence splits and we find that

$$
H_{0}^{\kappa}(X) \cong \widetilde{H_{0}^{\kappa}}(X) \oplus \mathbb{Z}
$$

The other statement is a trivial result.

\section{Properties of Euler characteristics}

In [7], Boxer, Karaca and Oztel have defined the Euler characteristics of digital images. Let $(X, \kappa)$ be a digital image of dimension $m$, and for each $q \geq 0$, let $\alpha_{q}$ be the number of digital $(\kappa, q)$-simplexes in $X$. The Euler characteristics of $X$, denoted by $\chi(X, \kappa)$, is defined by

$$
\chi(X, \kappa)=\sum_{q=0}^{m}(-1)^{q} \alpha_{q} .
$$

They also prove that if $(X, \kappa)$ is a digital image of dimension $m$, then

$$
\chi(X, \kappa)=\sum_{q=0}^{m}(-1)^{q} \operatorname{rankH}_{q}^{\kappa}(X) .
$$

We describe a few basic properties of the Euler characteristics. The Euler characteristics is a function $\chi$ which associates to each digital images $(X, \kappa)$ an integer $\chi(X, \kappa)$. The Euler characteristics is determined by the following properties which holds in Algebraic Topology. Let $X$ and $Y$ be any topological spaces, and $\{p\}$ be a single point set.

1. $\chi(\{p\})=1$.

2.If $X$ is homeomorphic to $Y$ then $\chi(X)=\chi(Y)$.

3.For any homotopic compact spaces $X$ and $Y$,

$$
\chi(X)=\chi(Y) .
$$

4.For every closed subset $C \subset X$,

$$
\chi(X)=\chi(C)+\chi(X \backslash C) .
$$

This property has a dual form

$$
\chi(X)=\chi(U)+\chi(X \backslash U),
$$

for every open subset $U \subset X$.

5. $\chi(X \times Y)=\chi(X) \cdot \chi(Y)$. 
The Euler characteristics of a single point digital image $\{p\}$ is $\chi(\{p\})=1$ since

$$
H_{q}^{\kappa}(\{p\})=\left\{\begin{array}{l}
\mathbb{Z}, q=0 \\
0, q \neq 0
\end{array}\right.
$$

In [7], Boxer et.al. prove the property (2). The other properties (3), (4) and (5) don't hold for digital images. We show by an example that the property (3) need not be hold for digital images.

Example 4.1. Let $X=M S C_{8}^{\prime}$ be digital image with 8 -adjacency and $Y=\{p\}$ be a single point digital image. Havana et.al. [1] show that

$$
H_{q}^{8}(X)=\left\{\begin{array}{l}
\mathbb{Z}, q=0,1 \\
0, q \neq 0,1
\end{array}\right.
$$

The Euler characteristics of $X$ is

$$
\begin{aligned}
\chi(X) & =\sum_{i=0}^{\infty}(-1)^{i} \operatorname{rank}_{i}^{8}(X) \\
& =1-1+0-0+\ldots \\
& =0 .
\end{aligned}
$$

Since

$$
H_{q}^{8}(Y)=\left\{\begin{array}{l}
\mathbb{Z}, q=0 \\
0, q \neq 0
\end{array}\right.
$$

we obtain

$$
\begin{aligned}
\chi(Y) & =\sum_{i=0}^{\infty}(-1)^{i} \operatorname{rankH}_{i}^{8}(Y) \\
& =1-0+0-\ldots \\
& =1
\end{aligned}
$$

As a consequence, although $X$ is $(8,8)$-homotopic to $Y$, $\chi(X) \neq \chi(Y)$. So the property (3) doesn't hold for digital images.

We can show that the property (4) needn't be hold for digital images.

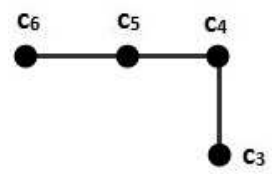

Fig. 4: $X \backslash C$

Example 4.2. Let $X$ be the digital image $M S C_{4}$. We take the digital projective line $\mathbb{Z} P^{1}$ which is the closed subset of $X$. Let this image be denoted by $C$. Consider a digital image $X \backslash C$. This is again the digital projective line $\mathbb{Z} P^{1}$ (see Figure 4). The Euler characteristics of $X, C$ and $X \backslash C$ are

$$
\chi(X)=0, \quad \chi(C)=1 \quad \text { and } \quad \chi(X \backslash C)=1,
$$

respectively. So we have

$$
\chi(X) \neq \chi(C)+\chi(X \backslash C) .
$$

Now we show by an example that the property (5) need not be hold for digital images.

Example 4.3. Let $X=[0,1]_{\mathbb{Z}}$ and $Y=[0,1]_{\mathbb{Z}} \times[0,1]_{\mathbb{Z}}$ be digital images with 2 and 4-adjacency, respectively. Consider a digital image

$$
X \times Y=[0,1]_{\mathbb{Z}} \times[0,1]_{\mathbb{Z}} \times[0,1]_{\mathbb{Z}}=M_{S S}^{\prime} .
$$

Boxer et.al. [7] show that

$$
H_{q}^{6}(X \times Y)= \begin{cases}\mathbb{Z}, & q=0 \\ \mathbb{Z}^{5}, & q=1 \\ 0, & q \neq 0,1\end{cases}
$$

The Euler characteristics of $X \times Y$ is (see [7])

$$
\begin{aligned}
\chi(X \times Y) & =\sum_{i=0}^{\infty}(-1)^{i} \operatorname{rankH}_{i}^{6}(X \times Y) \\
& =1-5+0-0+\ldots \\
& =-4
\end{aligned}
$$

On the other hand, since

$$
H_{q}^{2}(X)=\left\{\begin{array}{l}
\mathbb{Z}, q=0 \\
0, q \neq 0
\end{array} \quad \text { and } \quad H_{q}^{4}(Y)=\left\{\begin{array}{l}
\mathbb{Z}, q=0,1 \\
0, q \neq 0,1
\end{array}\right.\right.
$$

we have

$$
\begin{aligned}
\chi(X) & =\sum_{i=0}^{\infty}(-1)^{i} \operatorname{rankH}_{i}^{2}(X) \\
& =1-0+0-\ldots \\
& =1 .
\end{aligned}
$$

and

$$
\begin{aligned}
\chi(Y) & =\sum_{i=0}^{\infty}(-1)^{i} \operatorname{rankH}_{i}^{4}(Y) \\
& =1-1+0-0+\ldots \\
& =0 .
\end{aligned}
$$

As a result, we get

$$
\chi(X \times Y) \neq \chi(X) \cdot \chi(Y)
$$

\section{Conclusion}

The main goal of this study is to compute the relative homology groups and determine some properties of Euler characteristics for digital images. We hope that this article will be helpful in the study of digital homology groups and that will yield us to compute the homology groups of some part of the digital image when we know the homology groups of an entire image. 


\section{Acknowledgement}

The authors are grateful to the anonymous referee for a careful checking of the details and for helpful comments that improved this paper.

\section{References}

[1] H. Arslan, I. Karaca, and A. Oztel, Homology groups of $n$-dimensional digital images, XXI. Turkish National Mathematics Symposium, B1-13, (2008).

[2] L. Boxer, Digitally continuous functions, Pattern Recognition Letters, 15, 833-839 (1994).

[3] L. Boxer, A classical construction for the digital fundamental group, J. Math. Imaging Vis., 10, 51-62 (1999).

[4] L. Boxer, Properties of digital homotopy, Journal of Mathematical Imaging and Vision, 22, 19-26 (2005).

[5] L. Boxer, Homotopy properties of sphere-like digital images, Journal of Mathematical Imaging and Vision, 24, 167-175 (2006).

[6] L. Boxer, Digital products, wedges and covering spaces, Journal of Mathematical Imaging and Vision, 25, 169-171 (2006).

[7] L. Boxer, I. Karaca, and A. Oztel, Topological invariants in digital images, Journal of Mathematical Sciences: Advances and Applications, 11, 109-140 (2011).

[8] D. Diaz-Pernil, M.A. Gutierrez-Naranjo, P. Real, V. Sanchez-Canales, Computing Homology Groups in Binary 2D Imagery by Tissue-like P Systems, Romanian Journal of Information Science and Technology, 13, 141-152 (2010).

[9] O. Ege and I. Karaca, Fundamental properties of simplicial homology groups for digital images, American Journal of Computer Technology and Application, 1, 25-42 (2013).

[10] G.T. Herman, Oriented surfaces in digital spaces, CVGIP: Graphical Models and Image Processing, 55, 381-396 (1993).

[11] I. Karaca and O. Ege, Some results on simplicial homology groups of 2D digital images, Int. Journal of Information and Computer Science, 1, 198-203 (2012).

[12] E. Spanier, Algebraic Topology, McGraw-Hill, New York, (1966).

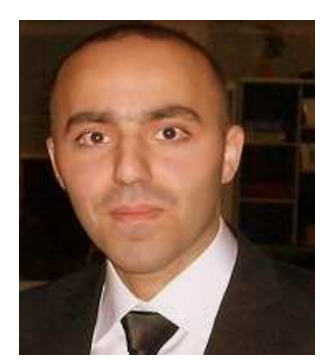

Ozgur Ege received a Bachelor's degree in Mathematics from Ege University in Turkey and started an integrated $\mathrm{PhD}$ programme in the field of algebraic topology at the same university. $\mathrm{He}$ is a research assistant at Celal Bayar University. His main research interests are digital topology, fixed point theory, algebraic topology, steenrod algebra and computer vision including the mathematical methods for digital images.

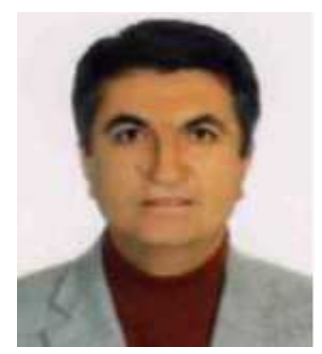

Ismet Karaca received a Bachelor's degree in Mathematics from Anadolu University in Turkey, a Master's in Mathematics from the university of Miami, and a $\mathrm{PhD}$ in Mathematics from Lehigh University. He is a Professor of Mathematics at Ege University in Izmir, Turkey. Dr. Karaca's research interests include homotopy theory, steenrod algebra, and digital topology. Prof. Karaca is a member of American Mathematical Society.

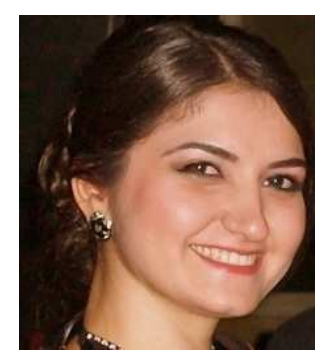

Meltem Erden Ege received a Bachelor's degree in Mathematics from Ege University in Turkey. She is currently a graduate student for a master programme in the field of algebraic topology at the same university. She is interested in algebraic topology, steenrod algebra

and digital topology. 